\title{
SUIZA COMO MODELO, SUIZA COMO TEMA. OBSERVACIONES PARA UN ANÁLISIS DEL CONCEPTO DE HEIMAT EN LA OBRA DE MAX FRISCH.
}

\section{Isabel Hernández.}

In this article the evolution of the Heimat concept in the work of the Swiss writer Max Frisch is analysed, highlighting its diverse aspects and, especially, the critical element which has influenced the literature written in German Switzerland throughout the $\mathrm{XX}^{\text {th }}$ century.

HEIMAT, die (Plural ungebräuchlich) Land, Landesteil od. Ort, in dem man [geboren u.] aufgewachsen ist od.sich durch ständigen Aufenthalt zu Hause fühlt (oft als gefïhlsbetonter Ausdruck enger Verbundenheit gegenüber einer bestimmten Gegend).

DUDEN. Das große Wörterbuch der deutschen Sprache.

Intentar un análisis del concepto de Heimat en la obra de un autor tan prolífico, complejo, apasionado, desarraigado y comprometido como Max Frisch, supone necesariamente entender este concepto, difícil por sus múltiples connotaciones, en diversas direcciones, si no totalmente diferentes entre sí, al menos sí diferentes en la elaboración o el tratamiento que de ellos hace el autor.

Una primera se orienta hacia el Estado suizo como entidad política y geográfica, con su situación peculiar en relación con el resto de los países europeos (su agrupación política mucho más temprana que la de cualquier otro país, sus cuatro lenguas oficiales amén de las múltiples variantes dialectales, su neutralidad o el secreto bancario, entre otros) ${ }^{1}$; una

\footnotetext{
1 "Die Schweiz ist oft als Ideal des fruchtbaren und friedlichen Zusammenlebens dreier Rassen, Sprachen und Kulturen gepriesen worden, sozusagen als kleiner Musterknabe für das grössere kommende Europa. Das ist gewiss schmeichelhaft für unser Land, aber nicht frei von einer idealisierenden Fernsicht. Die Wirklichkeit sieht von nahen etwas anders aus. Das Verhältnis der drei Sprachkreise ist keineswegs so problem- und spannungsfrei, wie es sich Aussenstehende vielleicht vorstellen. Dies gilt in besonderem Masse für die Welt der Literatur." H. Zbinden, "Aspekte schweizerischer Literatur im Verhältnis zu Europa". Vortrag an der europäischen Literaturtagung. Meran. NZZ (08.12.1963). Aunque la dualidad lengua hablada / lengua escrita supone un notable enriquecimiento para el escritor, el dialecto (al que los suizos siempre denominan como Mundart y nunca como Dialekt) no se utiliza apenas como lengua literaria, pues sustituir la lengua escrita por el dialecto representaría el aislamiento de las grandes culturas. De este modo, la situación cultural del país viene condicionada por- la
} 
segunda enmarca al escritor en su relación con la sociedad en la que vive, con la que convive, en la cual no siempre escribe, pero hacia la cual dirige su obra. Por último, el concepto de Heimat se extiende en la obra de Max Frisch, aunque con menor intensidad, a la relación que para el autor existe entre Suiza y el resto de los países de habla alemana, tanto en el terreno político como en el social o cultural, la relación, en definitiva, del propio Frisch con el mundo germanohablante, con el entorno lingüístico-cultural en el que se encuentra integrado todo escritor de lengua alemana.

A diferencia de Alemania o de Austria, en Suiza conviven ya desde antiguo tres lenguas oficiales, a las que se igualó el romanche en $1938^{2}$. Este modelo de Estado se mantiene con unas ideas políticas que en conjunto simbolizan la "idea del Estado suizo": libertad, neutralidad, democracia, federalismo y la idea republicana junto a la imagen de Suiza como portadora de misiones humanitarias especiales, Suiza como aproximadora de pueblos, como mediadora y representante de la paz.

Esta idea de Suiza, pues, como modelo de libertad, democracia y neutralidad, ha desempeñado un papel muy importante para el resto del mundo, especialmente en la época de la Ilustración, en diversos periodos del siglo XIX y durante las dos guerras mundiales. Pero aún más importante es el papel que esta idea ha desempeñado para los propios suizos. Durante el decenio de los años 30 de nuestro siglo, la acentuación de la defensa nacional se convirtió, al igual que en otros Estados neutrales, en una necesidad para demostrar a través de ella su voluntad y su afán de mantener esta neutralidad. Un aspecto de la que en Suiza se denominó como "geistige Landesverteidigung" fue el intento de despertar y reforzar precisamente la idea del Estado suizo, con su sistema democrático y libre. Más allá se trataba también de un intento de salvaguardar la historia y las tradiciones suizas. Aun cuando a lo largo de este decenio la ideología suiza alcanzó una cima histórica, esta mentalidad de defensa se siguió cuidando en ciertos círculos incluso después de la Guerra, y así, en los años 60, se seguía intentando aún mantenerla y revivirla. Sin embargo, este intento encontró una fuerte resistencia entre una joven generación de escritores que puso en tela de juicio la hasta entonces habitual concepción de Suiza como patria y con ella los conceptos de "libertad suiza" y "democracia". Fueron ellos los que colocaron a Suiza en el lugar en el que realmente se encuentra: no en el centro de Europa, sino completamente al margen, al margen de las grandes regiones lingüísticas, al margen de los bloques políticos. Y es precisamente la conciencia de esta situación marginal la que ha tomado cuerpo en la literatura suiza contemporánea, gracias a la toma de posición y a las múltiples reflexiones de los dos grandes escritores suizos del siglo XX: Max Frisch y Friedrich Dürrenmatt. Ellos fueron los primeros que se cuestionaron abiertamente los rasgos del carácter popular suizo

convivencia de lenguas oficiales y dialectos, y la falta de una cultura unitaria, a la que han contribuido no sólo la división lingüística, sino también la división religiosa y la falta de una capital representativa que hubiera podido hacer la función de centro cultural.

2 "In der Schweiz [...] haben wir es mit einer multidimensionalen Segmentierung zu tun; denn hier wird die Segmentierung ungefähr in gleicher Weise durch die Sprache, die Konfession, die soziale Schichtzugehörigkeit und die Region bestimmt. Diese Dimensionen überschneiden sich gegenseitig, so dass eine grosse Zahl von Subkulturen besteht." J. Steiner, Gewaltlose Politik und kulturelle Vielfalt. Hypothesen entwickelt am Beispiel der Schweiz, Haupt (Berna / Stuttgart 1970) 4. 
(amor a la libertad, independencia e individualismo, solidez, conservadurismo, amor a la tierra, materialismo, pequeñez y vanidad), y a ellos ha sucedido el planteamiento crítico que de este carácter han hecho generaciones posteriores de escritores ${ }^{3}$.

Haciendo un recorrido cronológico a lo largo de la producción de Max Frisch, podemos observar que en sus primeras obras no se deja traslucir ningún tipo de ideología política o social determinada. Tan sólo al finalizar la II Guerra Mundial se plantea el autor una "ideología suiza": comienzan a aparecer con mayor frecuencia reflexiones sobre el país y, aunque al principio se ocupa tan sólo de aspectos culturales, aparecen ya tratados conceptos como "libertad" o "democracia". Abundantes reflexiones al respecto se recogen en sus diarios y en la mayoría de sus colaboraciones en revistas y periódicos:

"Kein Zweifel, daß die Engländer und Amerikaner (die Russen kamen weniger in Frage) uns sofort beistehen würden gegen jeden Feind, der unsere Neutralität verletzt. Wir wußten, daß die Schweiz im Recht ist. Schweiz gleich Demokratie."

A medida que su producción va en aumento, la crítica del autor contra el país se hace más frecuente, más abundante: Frisch comienza a escribir poco a poco sobre las ideas tradicionales, sobre la política oficial o sobre los modelos de comportamiento social. Pero sin duda, el tema central de su producción literaria durante los primeros años lo constituye la figura del artista dentro de la sociedad: en las narraciones Jürg Reinhart (1934), Antwort

\footnotetext{
${ }^{3}$ El concepto "carácter popular" (Volkscharakter) ha desempeñado un papel importante como motivo literario y ha sido tratado por escritores, historiadores, periodistas, psicólogos y observadores tanto suizos como extranjeros. En el siglo XVIII, la imagen de Suiza y de su pueblo empezó a adquirir cierta importancia en la conciencia europea. Tres autores difundieron por Europa una imagen idílica del país alpino: Albrecht von Haller, Salomon Gessner y Jean Jacques Rousseau. Suiza se convirtió en modelo y fuente de inspiración para otros pueblos europeos durante más de medio siglo, y sólo gracias a tres elementos característicos del país: los Alpes, el amor a la libertad y la candidez de sus habitantes. El punto de partida lo constituyó el poema de Albrecht von Haller, Die Alpen (1729), en el que se refleja la belleza de las montañas, la pureza de la vida en ellas, la sencillez y la igualdad entre sus habitantes. Haller encontró un sucesor en Salomon Gessner, cuya obra Idyllen (1756) ratifica la imagen dibujada por Haller. Rousseau no desempeñó un papel importante sólo por sus descripciones del mundo de las montañas, sino también porque difundió a través de sus escritos su admiración por la Constitución y las instituciones políticas suizas. Los tres transmitieron al resto de los pueblos de Europa una imagen idílica y sentimental del pueblo suizo, viviendo en una especie de "candidez pastoril" como en la Arcadia.

Goethe también se sintió atraído por Suiza; sin embargo, en todo momento criticó duramente el anacronismo de su Constitución, basada en el mantenimiento del estado de las cosas en un momento en el que se estaban viviendo intensos cambios en todos los aspectos de la vida pública y privada. Con ello se adelantó en su crítica al periodo de declive político y social que Suiza sufrió a lo largo de la segunda mitad del siglo XVIII.

Durante el Romanticismo, la imagen de Suiza cambió, sobre todo en Alemania, a lo que contribuyó sin duda alguna el Wilhelm Tell de Schiller (1804), pues la imagen que este escritor —que curiosamente nunca había estado en Suiza - da aquí del país y de sus habitantes debió ser durante mucho tiempo no sólo determinante para la concepción europea, sino también para la opinión de los suizos sobre sí mismos, pues representaba la expresión adecuada de los sentimientos populares, y así, bien entrado ya el siglo XIX, autores como Gottfried Keller definen el carácter popular suizo tomando como punto de partida el concepto de libertad, clave en la obra de Schiller: "Der Nationalcharakter der Schweizer besteht nicht in den ältesten Ahnen noch in der Sage des Landes, noch, noch sonst in irgend etwas Materiellem; sondern er besteht in ihrer Liebe zur Freiheit, zur Unabhängigkeit, er besteht in ihrer außerordentlichen Anhänglichkeit an das kleine, aber schöne und teure Vaterland, er besteht in ihrem Heimweh, das sie in fremden, wenn auch den schönsten Ländern, befällt." G. Keller, Vermischte Gedanken über die Schweiz, G. Keller, Sämtliche Werke, Bd. 8 (Berlin 1958) 394 ss.
}

${ }^{4}$ M. Frisch, Dienstbüchlein, Suhrkamp (Frankfurt 1974) 77. 
aus der Stille (1937), en las novelas Die Schwierigen (1943) y Stiller (1954), así como en las obras de teatro Santa Cruz (1947) y Die chinesische Mauer (1947)5. En este mismo sentido escribe en sus diarios:

"Auch wenn es für den schweizerischen Künstler oft eine trockene Luft ist, was ihn in seiner Heimat umgibt, so ist dieses Übel, wie sehr es uns persönlich trifft, doch nur die leidige Kehrseite einer Haltung, die, von den meisten Deutschen als spießig verachtet, als ganzes unsere volle Bejahung hat - eben weil die gegenteilige Haltung, die ästhetische Kultur, zu einer tödlichen Katastrophe geführt hat, führen muß."

Aunque en un principio Frisch elige la forma teatral para ejercer su crítica social, es en la novela donde llegará a su cima y perfilará los rasgos de un estilo que ya jamás abandonará sus textos. Su primera obra, Jürg Reinhart, tiene como protagonista a un individuo de profesión liberal, un periodista que siempre se siente como un profano, que no participa de los hechos. Con ocasión de una estancia en el Mediterráneo (un espacio, por cierto, cargado de simbología en la obra de Frisch) retoma el hobby de su infancia, la pintura, y se dedica a seguir los caminos del mundo artístico. Die Schwierigen es la continuación de esta breve novela: el artista se ha establecido en Zúrich. Poco tiempo después decide, también de repente, dejar de pintar; quema algunas de sus pinturas y vende los marcos porque necesita dinero?

Anatol Ludwig Stiller es también un artista, un escultor mediocre, sin grandes éxitos. Al igual que Reinhart abandona un buen día su oficio de escultor, a su esposa, y junto a ellos, todo lo que había sido su vida hasta ese momento. Durante siete años intenta buscarle un nuevo sentido a su existencia. Cuando regresa a Suiza (ahora con el nombre de Mr. White) se niega a reconocer su vida anterior y su actividad artística. Tras ser identificado legalmente como Stiller, se retira al campo y se dedica a la producción de cerámica para turistas ("swiss pottery"). A lo largo de la novela, Stiller reflexiona sobre su actividad como artista: la desconfianza frente a la propia actividad creadora en un medio hostil al arte, y seguramente la desconfianza frente al arte en sí mismo, es el factor que le empuja a la ruptura.

¿Qué son estas dudas de Stiller sino las propias dudas de Frisch como artista, como escritor inmerso en un medio social predeterminado? ¿Puede un hombre dedicarse por completo al arte en ese entorno social? Seguramente no. Reinhart y Stiller son encarnaciones de la misma persona, pero en Stiller hay un elemento diferenciador: su

\footnotetext{
${ }^{5}$ La figura del artista comprometido con la sociedad tiene para Frisch sus antecedentes en la obra de Gottfried Keller Der grüne Heinrich. El protagonista de ésta, Heinrich Lee, se forma a sí mismo como pintor, pero se siente inseguro y aislado dentro de la sociedad y, tras años de estudio y de vagar por el mundo, regresa definitivamente a su hogar con una nueva visión de la sociedad. Abandona el arte y, en la segunda versión de la novela, consigue un puesto de funcionario en su ciudad natal. Frisch reconoce la influencia que en él ejerció la obra de Keller: "In jener Zeit las ich den Grünen Heinrich; das Buch, das mich seitenweise bestürzte wie eine Hellseherei, war natürlich der beste Vater, den man nur haben kann, [...]" M. Frisch, Tagebuch 1946-1949, Suhrkamp (Frankfurt 1985) 245.

${ }^{6}$ M. Frisch, Tagebücher 1946-1949, Suhrkamp (Frankfurt 1987) 289.

${ }^{7}$ Resulta inevitable ver aquí ciertos reflejos autobiográficos, pues Frisch decidió en una ocasión abandonar por completo la literatura y el periodismo, y quemó la mayor parte de sus apuntes y sus diarios.
} 
compromiso social. Por las mismas fechas en que se publicó la novela escribió Frisch el breve ensayo »achtung: die Schweiz« (1955): con energía, optimismo y entusiasmo, Frisch (igual que Stiller) pretende cambiar la sociedad en la que vive y lo expresa en voz alta, tanto en artículos de periódico, como en discursos públicos o novelas. Pero tan sólo un año después de la publicación de Stiller el pesimismo se ha apoderado del escritor. En su segunda versión de Die chinesische Mauer, publicada en 1955, reconoce que el artista no puede cambiar el mundo por medio de su arte. Frisch ha perdido la fe en la posibilidad de que el escritor pueda influir política y socialmente a través de su obra. Su compromiso se convierte en "anticompromiso" gracias a la ironía que utiliza cada vez con mayor frecuencia en sus discursos y en su producción periodística. Sin embargo, la separación entre ésta y su producción literaria resulta cada vez más clara: la crítica social queda relegada a la primera y perderá su sitio en el marco de sus textos literarios. Es el modo en el que Frisch, a pesar de sus múltiples dudas, mantiene su compromiso con la sociedad, tal vez únicamente por un fuerte sentimiento de responsabilidad hacia ella.

Este compromiso se refleja también en la convicción del autor de la importancia que tiene para los suizos la concepción de Suiza como resultado de una idea: "Dabei ist die Schweiz nicht anderes als eine Idee, die einmal realisiert worden ist." "Dos son los elementos que determinan esta "idea": libertad y democracia; ambos constituyen el núcleo que configura la práctica totalidad de la producción de Frisch. El concepto de "libertad" surge por primera vez en los apuntes de sus diarios: para él no existe una libertad absoluta, sino diversos grados de libertad y de falta de libertad, pero observa que, por lo general, el individuo se siente "libre" en una sociedad que protege sus propios intereses, y éste es exactamente el caso de Suiza'. Resulta curioso, sin embargo, que tanto libertad como democracia, que pasan por ser los bienes más amados de los suizos, no sean objeto de

\footnotetext{
${ }^{8}$ M. Frisch, »achtung: die Schweiz«, W. Obschlager (Hrsg.), Schweiz als Heimat? Suhrkamp (Frankfurt 1990) 133. Apuntes como éste encontramos constantemente en los diarios: “[...]; Kultur als eine Sache des ganzen Volkes; wir erkennen sie nicht allein auf dem Bücherschrank und am Flügel, sondern ebensosehr in der Art, wie man seine Untergebenen behandelt. Sofern man Kultur in diesem Sinne meint, der mir der zukünftige scheint, müßten wir in keiner Weise erschrecken, wenn sie uns gelegentlich einen Anachronismus nennen; ich meine weniger die Verwirklichung, sondern die Idee der Schweiz, die ich vor allem liebe, und wenn ich noch einmal aus freien Stücken wählen könnte, was die Geburt schon entschieden hat, möchte ich nichts anders als ein Schweizer sein; nach der Idee, die unsere eigentliche Heimat ist, sind es natürlich auch einzelne Landschaften, die man liebt, aber erst in zweiter Linie; am wenigsten weiß ich, ob ich unsere Landsleute liebe — sicher nicht mehr als die entsprechenden Gesichter aus anderen Völkern, und es erschiene mir nicht einmal als Ziel, im Gegenteil; Liebe zum Vaterland, so verstanden, wird zum Verrat an der Heimat; unsere Heimat ist der Mensch; ihm vor allem gehört unsere Treue; daß sich Vaterland und Menschheit nicht ausschließen, darin besteht ja das große Glück, Sohn eines kleinen Landes zu sein." M. Frisch, Tagebuch 1946-1949, Suhrkamp (Frankfurt 1985) 149 ss.

${ }^{9}$ Stiller tiene como núcleo central precisamente este concepto. Bohnenblust y Stiller discuten al respecto: "PS. Gegenüber der Tatsache, daß die Schweiz nicht nur ein kleines Land ist, sondern durch den Lauf der Welt immer noch kleiner wird, hat mein Verteidiger überhaupt keinen Humor. [...] »Die Größe eines Landes «, sagt er, »das ist nicht als Fläche zu messen und nicht als Einwohnerzahl; die Größe unseres Landes ist die Größe seines Geistes.« [...] Als Häftling, mag sein, bin ich besonders empfindlich auf ihr Schlagwort von der Freiheit. Was, zum Teufel, machen sie denn mit ihrer sagenhaften Freiheit? [...] Wahrscheinlich kann es überhaupt keine Freiheit geben, wie man sie hierzulande zu haben behauptet; es gibt nur Unterschiede in der Unfreiheit, ... [...]. Sie wagen es unter keinen Umständen, sich selbst in Zweifel zu ziehen. Ist das nicht gerade das Zeichen geistiger Unfreiheit? [...] Aber was hat all das zu tun mit Freiheit? Ich sehe doch ihre Gesichter; sind sie frei?" M. Frisch, Stiller, Suhrkamp (Frankfurt 1985) 149 ss.
} 
manifestaciones públicas: hablar de libertad o de democracia supone seguramente atentar contra ellas, y de este modo, el análisis que Frisch hace de ambas no es en el fondo más que un enfrentamiento con el propio país, con Suiza ${ }^{10}$. Constantemente propone la necesidad de alternativas políticas y, sobre todo, la necesidad de una oposición, sin obtener, claro está, ningún resultado.

Es en relación con estos dos conceptos como Frisch comienza a analizar la idea de Heimat. El término "patria" (Vaterland) y una reflexión sobre la misma aparece por primera vez en Blätter aus dem Brotsack, escrita en el otoño de 1939 mientras servía como soldado:

"Wir werden geboren und haben nicht um unser Leben gebeten, nicht unser Vaterland erwählt. Einmal am Leben aber, ja, wie hängen wir daran, und wie lieben wir auch das Land, das unser Vaterland ist, selbst wenn es nicht in aller Mund wäre, selbst wenn es uns schmerzt. Wir haben Menschen gesehen, denen man das Vaterland aus der Seele gerissen hatte, so daß sie langsam daran verbluten. Aber auch wir, die wir eine Fahne haben, einen Flecken auf der Erde, wo uns nur das Gewissen gebietet, müssen eine letzte Heimat erst suchen, und wer weiß, ob sie auf dieser Erde ist? Wir wollen das Grenzenlose, ob man es Herrgott nennt oder anders, nicht preisgeben und aus dem Boden, den es uns lieh, niemals einen Götzen machen, der den Menschen in uns erwürgt; wir werden unser Vaterland lieben und es verteidigen, niemals es anbeten."

Hay en esta nota una clara toma de conciencia, aunque en tono reservado. Heimat tiene en este pasaje un acento casi metafórico, que pronto perderá para convertirse en una constante en los textos de Frisch, ya sea con breves alusiones, ya sea tratada abiertamente. ¿Por qué esta continua preocupación? ¿Cuál es el motivo de la crítica constante contra su país? ¿Le ocurre sólo a él o es una característica común al resto de los escritores suizos? La respuesta no se encuentra en otra parte más que en la relación de amor y odio que el suizo tiene respecto de su país:

"Heimat. Die Summe unserer Sitten und Unsitten, eine gewisse Gewöhnung, das Gemeinsame einer gleichen Umgebung, all das ist nicht wertlos. Am gleichen Ufer gespielt zu haben, natürlich hat es etwas Verbindendes; es für Verwandschaft anzusehen, wäre ein Irrtum, der uns früher oder später, indem wir ihn nur als Enttäuschung erleben und nicht als Irrtum erkennen, ungerecht macht. Heimat ist unerläßlich, aber sie ist nicht nur an Ländereien gebunden. Heimat ist der Mensch, dessen Wesen wir vernehmen und erreichen. Insofern ist sie vielleicht an die Sprache gebunden. Vielleicht; denn in der Sprache allein ist sie ja nicht. Worte verbinden nur, wo unsere Wellenhängen übereinstimmen; das Wiederum heißt nicht Einverständnis, das es nirgends so häufig gibt wie unter Wesensfremden die einander mißdeuten, sondern Erreichbarkeit, und gerade wo man sich unter anderen

\footnotetext{
${ }^{10}$ También en Stiller hay una reflexión sobre la democracia suiza en relación, claro está, con el tema central de la obra, la libertad: "Sturzenegger sagt nur: Wir sind eine Demokratie! Ich verstehe ihn nicht. Worin bestünde denn die Freiheit einer demokratischen Verfassung, wenn nicht eben darin, daß sie dem Volk immerfort das Recht gibt, seine Gesetze im demokratischen Sinn zu verändern, wenn es nötig ist, um sich in einem veränderten Zeitalter behaupten zu können?" M. Frisch, op.cit., pág. 248.

${ }^{11}$ M. Frisch, Blätter aus dem Brotsack, W. Obschlager, op.cit., 14.
} 
Bedingungen trifft, erleben wir, durch keine gleichen Gewöhnungen getäuscht, das Verwandte oft um so reiner, um so überraschender und um so dankbarer, um so fruchtabarer." 12

Se acepta la idea de una Heimat propia a la vez que se rechazan determinadas formas de manifestación del amor hacia ella. Por una parte, la Heimat resulta ser algo ilimitado, que no tiene ni puede tener fronteras; pero, por otra, es la persona "dessen Wesen wir vernehmen und erreichen", y además, "an die Sprache gebunden", lo cual es ya de por sí una gran limitación ${ }^{13}$.

Las primera críticas expresas en un tono ciertamente duro son consecuencia de su viaje a América en 1951. El distanciamiento, unido a nuevos puntos de vista lo hacen posible. Stiller es el resultado de sus años en ese gran país: la novela comienza transmitiendo la idea de que Suiza es una cárcel y Stiller (Frisch) es el encargado de criticar todo aquello que tradicionalmente ha caracterizado y caracteriza al Estado, a las instituciones y al pueblo suizo $^{14}$. El protagonista se siente totalmente incapaz de vivir en la sociedad en la que ha nacido, tal vez por incapacidad suya o tal vez porque la sociedad en sí es falsa ${ }^{15}$. Es una sociedad que no quiere avanzar hacia el futuro con el resto de Europa, sino que prefiere

\footnotetext{
${ }^{12}$ M. Frisch, Tagebuch 1946-1949, Suhrkamp (Frankfurt 1987) 354.

${ }^{13} \mathrm{La}$ asociación Heimat-lengua materna es una de las más básicas y de las que se confirman en todo análisis sobre este concepto. Frisch se reconoce a sí mismo en todo momento como un escritor suizo y defiende siempre las variedades linguísticas y dialectales del país, pues hablándolas es como uno se reconoce a sí mismo como perteneciente a un grupo o a una comunidad determinada, a un suelo concreto y específico: "Obschon die Deutschschweizer, ausgenommen Schriftsteller und vielleicht Pfarrer, sich nur in der Mundart wohlfühlen, [...] Jeder Mann hat die Volksschule besucht und versteht das Hochdeutsch, [...] ohne Mühe." M. Frisch, Dienstbüchlein, Suhrkamp (Frankfurt 1974) 16.

El dialecto es para los escritores suizos una lengua inconsciente, el Hochdeutsch, la lengua estándar, es una lengua consciente, es decir, que el escritor habla en dialecto (manifestación del acto inconsciente del habla), pero cuando escribe (manifestación del acto consciente de elaboración de un texto escrito) lo hace en la lengua estándar. El hecho de que el alemán estándar que utilizan los escritores suizos esté plagado de helvetismos no quiere decir que no sean capaces de separar el dialecto de la lengua estándar. El uso de helvetismos es consciente, pues de él resulta un enriquecimiento de la lengua, que el escritor agradece en todo momento: "DANKBARKEITEN [...] p. die Spannung zwischen Mundart und Schriftsprache." M. Frisch, Tagebuch 19661971, Suhrkamp (Frankfurt 1989) 255. En este mismo sentido véase también W. Schenker, Mundart und Schriftsprache, W. Schmitz, Max Frisch, Suhrkamp (Frankfurt 1987) 47-59.

${ }_{14}$ "[...], daß es Gerechtigkeit gebe zumindest in einem Rechtstaat, zumindest in der Schweiz. [...] »Ich verstehe sie vollkommen«, sagt er, »ich verstehe Sie vollkommen! Sie sind ungehalten über die Schweiz, die Sie mit Untersuchung empfängt, begreiflicherweise, ich meine: begreiflicherweise ungehalten, denn es ist bitter, die Heimat durch ein Gitter zu sehen- «Was heißt Heimat?« frage ich.” M. Frisch, Stiller, Suhrkamp (Frankfurt 1988) 21-22.

15 “[...]: wie sauber sie hierzulande bauen, wie sicher, wie schmuck, wie gediegen, wie seriös, wie makellos, wie gewissenhaft, wie geschmackvoll, wie gepflegt, wie gründlich, wie ernsthaft und so weiter, alles für die Ewigkeit. [...] Architekten voll Talent und Heimatliebe bauen, wie ich neulich gesehen habe, Geschäftshäuser im ungefähren Maßstab des sechzehnten oder siebzehnten oder achtzehnten Jahrhunderts. [...], zukünftig zu sein in einem Volk, das eigentlich, wie mir scheint, nicht die Zukunft will, sondern die Vergangenheit. Hat die Schweiz (so frage ich Sturzenegger) irgendein Ziel in die Zukunft hinaus? [...] Damals hatte die Schweiz eine geschichtliche Gegenwart. Hat sie das heute? Das Heimweh nach dem Vorgestern, das die meisten Menschen hierzulande bestimmt, ist bedrückend. Es zeigt sich [...] in der Literatur: die meisten und wohl auch besten Erzählungen entführen in die ländliche Idylle; das bäuerliche Leben erscheint als letztes Reduit der Innerlichkeit; [...]" M. Frisch, Stiller, 244 ss.
} 
seguir viviendo en el pasado, en su situación de isla en medio de Europa, en su situación neutral.

El contraste con América produce esta reacción en el escritor: Estados Unidos se convierte para él en su segunda Heimat, y allí pasa largas temporadas (1951, 1956, 1970, 1971). En 1953 publica un artículo titulado »Unsere Arroganz gegenüber Amerika« en el que defiende la forma y el modelo de vida americanos frente a la "arrogancia europea" y a "nuestros prejuicios". Estas impresiones se plasman también en el discurso pronunciado en Zúrich ese mismo año y publicado con el título »Cum grano salis«, en el que se reflejan las sensaciones que le produce el regreso a Suiza tras su larga estancia en Estados Unidos y Méjico $^{16}$. La insistencia en la cuestión suiza llega a ser tal que se manifiesta por igual, con textos prácticamente idénticos, tanto en el mencionado discurso como en Stiller: ${ }^{17}$

"Haben wir eine schöpferische Hoffnung? Unsere letzte grosse, wirklich lebendige Epoche war die Mitte des 19. Jahrhunderts, die Achtundvierziger Jahre; damals hatten sie einen Entwurf, sie wollten, was es zuvor noch nicht gegeben hatte, und freuten sich auf das Übermorgen; damals hatte die Schweiz eine geschichtliche Gegenwart. Haben wir sie heute? Das Heimweh nach dem Vorgestern, das die meisten Schweizer zu bestimmen scheint, sehen wir allenthalben [...]" ("Cum grano salis")
"Haben wir eine schöpferische Hoffnung? Ihre letzte große und wirklich lebendige Epoche (laut Vorträgen meines Verteidigers) war die Mitte des neunzehnten Jahrhunderts, die sogenannten Achtundvierziger-Jahre. Damals hatten sie einen Entwurf. Damals wollten sie, was es zuvor noch nie gegeben hatte, und freuten sich auf das Morgen, das Übermorgen. Damals hatte die Schweiz eine geschichtliche Gegenwart. Hat sie das heute? Das Heimweh nach dem Vorgestern, das die meisten Menschen hierzulande bestimmt, ist bedrückend." (Stiller)

Lentamente se ha ido produciendo un cambio y la crítica de Frisch se ha ido haciendo cada vez más dura, más feroz; Heimat ha ganado terreno a Vaterland, que adquiere con el tiempo connotaciones peyorativas, y del mismo modo, aunque con unos tonos completamente diferentes, también el concepto de Heimat irá cambiando de significado continuamente en su obra.

Uno de los ámbitos en los que se centra su crítica es en la pequeñez del país: partiendo de la pequeñez física, geográfica, de la conocida como "Enge der Schweiz"18, critica la

\footnotetext{
16 " [...] der Heimkehrende wird kaum einen schweizerischen Bau treffen, wo ihn nicht reihenweise etwa die folgenden Beiwörter einfallen: Schmuck, gediegen, gründlich, gepflegt, geschmackvoll, sicher, sauber, gepützelt, makellos, seriös, sehr seriös." M. Frisch, "Cum grano salis". M. Frisch, Gesammelte Werke, Bd. 5, Suhrkamp (Frankfurt 1976) 230.

${ }^{17}$ Vid. respectivamente M. Frisch, "Cum grano salis", op.cit., 236; M. Frisch, Stiller, Suhrkamp (Frankfurt 1973) 247-248.

${ }^{18}$ Precisamente en este sentido véase la obra de Paul Nizon, Diskurs in der Enge, Suhrkamp (Frankfurt 1990), así como sus declaraciones en un encuentro sobre novela europea celebrado en Madrid en enero de 1993: " «No
} 
pequeñez mental del ciudadano suizo, su falta de miras, su temor ante todo lo que signifique un cambio $^{19}$, de tal modo que llegados a este punto, central y decisivo para el análisis de este complejo concepto, podemos formularnos aquí la misma pregunta que Frisch formula una y otra vez, y que, en realidad, resulta ser el punto de partida del cual arranca la totalidad del elemento crítico presente no sólo en su obra, sino también en la de Dürrenmatt y en la de generaciones posteriores de escritores suizos: ¿Qué es en realidad Suiza? Simplemente un mito: el mito de la libertad, de la neutralidad, del bienestar en medio del continente europeo:

"Wir sind eine Insel, und man sagt, Europa braucht eine Insel. Wer sagt es? Wir sagen es." ${ }^{20}$

"Wir Schweizer wissen ja, daß wir offiziell im Weltgeschehen nirgends eingreifen. Womit wir zu tun haben, ist der Welthandel." ${ }^{21}$

"Vermögen? Eine verfassungswidrige Frage; sie verletzt das schweizerische Bankgehimnis, dem dieses Land soviel verdankt, [...]"22

Frisch insiste en combatir estos clichés, estos mitos y en sus textos dedica a la Heimat innumerables pasajes a lo largo de los cuales lleva a cabo un análisis de la misma desde todos los puntos de vista posibles, desde todas las perspectivas imaginables: política (refiriéndose a temas como democracia, libertad, neutralidad), cultura (situación del escritor en Suiza, compromiso social, Estado y relación de éste con el mundo cultural), relaciones de Suiza con el extranjero, y un largo etcétera ${ }^{23}$. Suiza debe desempeñar un papel activo y

existen grandes ciudades en Suiza. No hay ciudades que recojan el mundo entero, que puedan considerarse metrópolis», señala el escritor y sigue hablando por el protagonista de su novela, que huye de su país para salir al mundo, para conocer la vida. «No creo que Suiza esté sólo fuera del mundo, sino también de la historia - prosigue Nizon-. Es como una isla, como una gruta o un sótano que se ha quedado fuera del universo en que las cosas se mueven, se transforman y cambian.»" E. Rodríguez, "«Europa no puede convertirse en una fortaleza», afirma Paul Nizon", El Mundo (20.01.1993) 46.

${ }^{19}$ Dos fragmentos reproducen fielmente esta concepción:

"Basel, März 1946 [...] Wie klein unser Land ist. Unser Sehnsucht nach Welt, unser Verlangen nach den großen und flachen Horizonten, nach Masten und Molen, nach Gras auf den Dünen, nach spiegelnden Grachten, nach Wolken über dem offenen Meer; unser Verlangen nach Wasser, das uns verbindet mit allen Küsten dieser Erde; unser Heimweh nach der Fremde -“M. Frisch, Tagebuch 1946-1949, Suhrkamp (Frankfurt 1987) 22;

"Ein kleines Herrenvolk sieht sich in Gefahr: man hat Arbeitskräfte gerufen, und es kommen Menschen. Sie fressen den Wohlstand nicht auf, im Gegenteil, sie sind für den Wohlstand unerläßlich. Aber sie sind da. Gastarbeiter oder Fremdarbeiter? [...] Man ist kein Rassist; es ist schließlich eine Tradition, daß man nicht rassistisch ist, [...] Die andere Seite ist uns bekannt: der Mythos, den die Schweiz sich selber gibt, und die Tatsache daß der Mythos keine Probleme löst; daher die Hysterie der Hilflosigkeit; jedes Problem, das wir selbst zu bewältigen haben, schickt den Begriff der Schweiz in die Reparatur. Hoffentlich gelingt sie." M. Frisch, "Überfremdung I", W. Obschlager, op.cit., 104.

${ }^{20}$ M. Frisch, Blätter aus dem Brotsack, W. Obschlager, op.cit., 104.

${ }^{21}$ M. Frisch, "Und die Schweiz? Ein Interview", W. Obschlager, op.cit., 209.

${ }^{22}$ M. Frisch, Mein Name sei Gantenbein, Suhrkamp (Frankfurt 1987) 41.

${ }^{23} \mathrm{El}$ intento de combatir estos clichés se expresa en pasajes como el siguiente: "Wir wollen die Schweiz nicht als Museum, als europäischer Kurort, als Altersasyl, als Paßbehörde, als Tresor, als Treffpunkt der Krämer und Spitzel, als Idylle; sondern wir wollen die Schweiz als ein kleines, aber aktives Land, das zur Welt gehört. Wir wollen kein schweizerisches Minderwertigkeitsgefühl, keinen schweizerischen Größenwahn; sondern wir wollen eine Schweiz, die sich selbst ins Gesicht zu schauen wagt, eine Schweiz, die sich nicht vor der Wandlung scheut, 
participar en los acontecimientos europeos y mundiales; no es un paraíso idílico, sino un país moderno que debe mirar hacia el futuro y no hacia el pasado, hacia los mitos de su historia ${ }^{24}$.

A pesar de la decepción sufrida por Frisch al comprobar que las duras críticas ejercidas en sus textos no tenían la repercusión deseada, la actitud que tanto él como Dürrenmatt adoptaron respecto a su país ha tenido su continuación en las posteriores generaciones de escritores suizos. Baste comprobar textos como los publicados recientemente por Adolf Muschg en su volumen recopitalorio Wenn Auschwitz in der Schweiz liegt $t^{25}$, o los resultados del boicot que llevaron a cabo escritores e intelectuales suizos contra la celebración del $700^{\circ}$ Aniversario de la fundación de la Confederación Helvética ${ }^{26}$. En Frisch, sin embargo, y como es natural en una obra que se extiende a lo largo de más de cincuenta años, el enfrentamiento con la realidad que late tras el concepto de Heimat supone una clara evolución en la reflexión sobre un tema tan complejo y tan arraigado en la tradición literaria de lengua alemana como lo es éste. Ningún escritor suizo ha dejado una producción literaria en la que este concepto haya marcado su obra de principio a fin como Max Frisch, de ahí que en tan extensa producción se observen con claridad dos grandes bloques: el primero tiene como centro a Suiza, al país en su totalidad, y aparece desde los primeros momentos hasta bien entrados ya los años 60. En él pueden observarse a su vez tres fases perfectamente diferenciadas:

1. durante los primeros años encontramos en sus textos una visión idealizada de la Heimat, de Suiza en general, aquella misma visión que tenían los escritores del siglo XIX, según la cual, Suiza había de servir como modelo a otros países, debido sobre todo a su sistema político que permite libertad y democracia para todos;

2. durante los años de la guerra y la inmediata posguerra, Frisch elude el tema o hace tan sólo brevísimas menciones a él;

3. a partir de los años 50 la visión idealizada desaparece y surge un punto de vista crítico que se acentuará durante los años 60: la validez de los conceptos "democracia" y "libertad", entre otros, se cuestionan constantemente.

El segundo gran bloque se centra en la Heimat local, no ya en el país, sino en la "kleine Heimat"; se produce una reducción espacial y Frisch pasa de concebir como Heimat el país

eine Schweiz, die ihre Idee an den heutigen Problemen und mit den heutigen Mitteln zu verwirklichen sucht." M. Frisch, »achtung: die Schweiz«, W. Obschlager, op.cit., 149.

${ }^{24}$ En este sentido, Frisch desmitifica la figura del héroe popular, Guillermo Tell, en su obra Wilhelm Tell für die Schule (1970), del mismo modo en que constantemente intenta desmitificar todo lo referente a tradición e historia en la mayoría de sus textos: "[...], so kann ich mich allerdings, wenn ich HEIMAT sage, nicht mehr begnügen mit Pfannenstiel und Greifensee und Lindenhof und Mundart, nicht einmal mit Gottfried Keller; dann gehört zu meiner Heimat auch die Schande, zum Beispiel die schweizerische Flüchtlingspolitik im Zweiten Weltkrieg und anderes, was zu unserer Zeit geschieht oder nicht geschieht [...] so verstehe ich mich als Schweizer ganz und gar dieser meiner Heimat verbunden —einmal wieder - in Zorn und Scham.” M. Frisch, "Die Schweiz als Heimat?", W. Obschlager, op.cit., 373.

${ }^{25}$ A. Muschg, Wenn Auschwitz in der Schweiz liegt, Suhrkamp (Frankfurt 1997).

${ }^{26}$ Sobre el boicot cultural véase F. Lerch / A. Simmen (Hrsg.), Der leergeglaubte Staat. Kulturboykott: Gegen die 700-Jahr-Feier der Schweiz, Rotpunkt (Zürich 1991). 
donde ha nacido, a concebir ahora como tal aquel territorio, espacio geográfico, e incluso personas, que el autor identifica como suyos, como su auténtica Heimat. Esta nueva consideración empieza a reflejarse en los textos escritos tras los acontecimientos políticos y sociales del 68 y se irá acentuando cada vez más hasta sus últimas obras, debido, entre otras cosas, al desengaño en cuestiones políticas y sociales, por un lado, y al paso inevitable de los años, por otro. Este último periodo creativo de Frisch se caracteriza precisamente por una actitud retrospectiva, por un marcado interiorismo, por la reflexión sobre el tiempo pasado, por el hombre como tal ${ }^{27}$. Las descripciones de paisajes se hacen ahora mucho más frecuentes, y Frisch se recrea en ellas hasta en el más mínimo detalle cuando pinta algún rincón de un valle recóndito o de una ciudad cualquiera de la pequeña geografía suiza, una geografía que está cambiando y a la que se está poniendo en peligro con estos cambios. Son ahora los lugares y las personas que viven en este bello entorno amenazado los que centran su atención:

"Ein Tal ohne Durchgangsverkehr.

Hin und wieder hört man den flatternden Schall eines Helikopters, der Baustoff transportiert, irgendwo wird noch gebaut.

Sonst ereignet sich wenig.

Früher hat die Bevölkerung von der Strohflechterei gelebt, Heim-Industrie mit Kinderarbeit, bis auf dem Markt zu Mailand die billigen Japaner erschienen sind.

Die Jungen wandern aus.

Ein Stausee ist nicht vorgesehen.

Einen Einheimischen zu finden, der einem Rentner das Gras mäht, ist schon beinahe möglich. Auch das Gras lohnt sich nicht mehr. Trotzdem steigen die Bodenpreise auch hier; wer Boden besitzt, auch wo er sich nicht lohnt, fühlt sich sicherer. Die Feigen werden nicht reif, aber die Trauben. Viele Kastanien haben den Krebs. Im Herbst sind Holzfäller an der Arbeit, Tage lang hört man das Geknatter ihrer Motorsäge, ohne die Männer im Gehölz zu sehen.

Alles in allem ein stilles Tal." ${ }^{28}$

\footnotetext{
${ }^{27}$ La predisposición con la que Frisch escribe ahora sus textos queda recogida en la nota de advertencia que el propio autor hace a los lectores de Montauk: "DIES IST EIN AUFRICHTIGES BUCH, LESER, ES WARNT DICH SHCON BEIM EINTRITT, DASS ICH MIR DARIN KEIN ANDERES ENDE VORGESETZT HABE ALS EIN HÄUSLICHES UND PRIVATES... ICH HABE ES DEM PERSÖNLICHEN GEBRAUCH MEINER FREUNDE UND ANGEHÖRIGEN GEWIDMET, AUF DASS SIE, WENN SIE MICH VERLOREN HABEN, DARIN EINIGE ZÜGE MEINER LEBENSART UND MEINER GEMÜTSVERFASSUNG WIEDERFINDEN... DENN ICH BIN ES, DEN ICH DARSTELLE. MEINE FEHLER WIRD MAN HIER FINDEN, SO WIE SIE SIND, UND MEIN UNBEFANGENES WESEN, SO WEIT ES NUR DIE ÖFFENTLICHE SCHICKLICHKEIT ERLAUBT... SO BIN ICH SELBER, LESER, DER EINZIGE INHALT MEINES BUCHES; ES IST NICHT BILLIG, DASS DU DEINE MUSSE AUF EINEN SO EITLEN UND GERINGFÜGIGEN GEGENSTAND VERWENDEST." M. Frisch, Montauk, Suhrkamp (Frankfurt 1981) 5.

${ }^{28}$ M. Frisch, Der Mensch erscheint im Holozän, Suhrkamp (Frankfurt 1981) 62-63. O también descripciones como la siguiente: "Er schenkte mir das Engadin. Noch heute kann ich nicht durch jene Gegend fahren, ohne an W. zu denken. Und ich meine ja nicht nur, daß die Reise ins Engadin für mich unerschwinglich gewesen wäre. Er kannte das Engadin. Er war auch der bessere Alpinist. Seine Familie hatte dort einen Bergführer, der ihn Jahr für Jahr unterrichtet hatte. Ohne W. wäre ich nie auf diese Berge gelangt. Er wußte, wo und wann Lawinen drohen und wie man sich in einem bedenklichen Gelände zu verhalten hat; er knüpfte die rote Lawinen-Schnur an seinen
} 
En este punto, en la concepción de lo local como Heimat, y en la recreación literaria de lo pequeño, de lo conocido como espacio vital necesario, como espacio propio, es donde Frisch conecta con la joven generación de escritores que nace en Suiza a lo largo del decenio de los 60 y que, en cierto modo, encuentra su motivación y sus fuentes de inspiración en las ideas, en los textos de Frisch y Dürrenmatt, a pesar de que en ninguno de ellos se manifiesta tan abiertamente la carga social y política presente en las obras de los dos anteriores. Aunque comparten con ellos la crítica de la idea de Suiza como mito, resulta evidente que no conciben Suiza como Heimat, pues para ellos este concepto se ha reducido a lo local ${ }^{29}$. Esta reducción implica una mayor apreciación de las variantes dialectales y un retorno a la literatura en dialecto (tan sólo apreciable en la lírica) y a un mayor uso de estas variantes en los textos en prosa. ${ }^{30}$

La tercera y última dirección hacia la que se orienta la consideración del concepto de Heimat es la ya apuntada en relación con el resto de los países de lengua alemana. En la primavera de 1935, Frisch visitó Alemania por primera vez. Las impresiones que anota en su Kleines Tagebuch einer deutschen Reise (1935) son más de tipo cultural que político y dejan patente la sensación de una fuerte interrelación cultural entre ambos países. Diez años más tarde vuelve a recrear en otra obra la presencia del país vecino, un país, en esta ocasión, destruido por la guerra: es la obra de teatro Nun singen sie wieder (1946). El escritor suizo se acerca al tema con moderación, con la moderación propia del que ha permanecido ajeno al conflicto y no lo ha visto con sus propios ojos. Pero el motivo, recurrido y recurrente, de la culpabilidad, se vislumbra ya en este primer acercamiento a la realidad que se ha vivido de espaldas. No transcurrirá mucho tiempo hasta que Frisch comience a acentuar en sus artículos y ensayos la necesidad de ocuparse de la cuestión alemana, tanto por motivos fronterizos como por ser dos pueblos unidos por una lengua común. Y así, poco a poco, se irá desenmascarando la culpabilidad, no sólo la alemana, sino también la suiza, cuyo máximo exponente literario es, sin duda alguna, Stiller ${ }^{31}$. Frisch no descarta en absoluto la posibilidad de otro futuro régimen nacionalsocialista y adopta una cierta actitud de desconfianza ante la Alemania de Aderauer, de cuya democracia duda;

Rucksack, betrachtete gewissenhaft den Hang und prüfte den Schnee, dann sauste er voran in die Tiefe, und ich hatte mich nur an seine kühne Spur zu halten, so gut ich's halt konnte." M. Frisch, Montauk, Suhrkamp (Frankfurt 1981) 36-37.

${ }^{29}$ En este sentido véase M. I. Hernández, “¿Sigue siendo Suiza un tema para sus escritores? Nuevas tendencias en la narrativa suiza actual”, Anuari de Filologia A 5 (1994) 73-80.

${ }^{30} \mathrm{La}$ obra de Frisch está repleta de reflexiones sobre la importancia del dialecto para el escritor suizo: "Mundart. Es war irgendwo am Mittelmeer. Er traf auf schweizerische Landsleute, und seit soundsovielen Monaten war es das erstemal, daß er wieder sein Schweizerdeutsch mundhabte. Seine deutschen Begleiter waren etwas verletzt, sobald er sich dieser Geheimsprache bediente, und wehrten sich einfach, indem sie feststellten, daß es eine häßliche Sprache wäre.” M. Frisch, Stichworte (Hrsg. von Uwe Johnson), Frankfurt (Suhrkamp 1985) 61.

31 "»Es ist komisch«, fand Sibylle, »wie böse du jedesmal wirst, wenn du von der Schweiz redest! [...] »Warten wir ab«, sagte er un stellte die Wasserpfanne auf, »bis Deutschland, unser tüchtiger Nachbar, wieder das große Geschäft ist! Uns wenn die es nochmals mit Faschismus versuchen, an der Schweiz wird's nicht fehlen, sie wird sekundieren. [...] Genau wie damals! Bis der freundliche Nachbar unseren Käse nicht mehr frißt oder unsere Uhren nicht braucht, weil die Zeit fortan nach seinen Uhren geht, dann das große Geschrei, o ja, das Ende der Freiheit, das Ende des Geschäftes, dannplötzlich sind wir wieder der ewige Hort der Humanität, wie immer, die Inhaber des Friedens, die Priester des Rechts - zum Kotzen«, sagte Stiller, »du entschuldigst, aber es ist so.« M. Frisch, Stiller, Suhrkamp (Frankfurt 1988) 266. 
pero al mismo tiempo duda también de la conciencia suiza (Biedermann und die Brandstifter, 1958; Andorra, 1961). De este modo, la cuestión alemana, el reconocimiento de la necesidad de ocuparse del espacio geográfico que, a su vez es el gran tronco cultural en torno al cual se agrupan todos los países de habla alemana, le sirve de gran plataforma para atacar, desde otra perspectiva, el gran tema de la obra de Frisch: Suiza.

Este es el marco en el que se ha desarrollado la literatura suiza escrita en lengua alemana del siglo XX. No pueden hacerse referencias a ella, sin entender la huella que la obra de Frisch ha dejado tanto en sus contemporáneos, como en los que en generaciones posteriores le tuvieron como modelo, puesto que la preocupación por la cuestión suiza, que él impulsó hasta las cotas más altas, ha sido el tema, el motivo en torno al cual ha despertado la vida literaria en la Suiza alemana. El siglo XX se cierra de este modo con el análisis y la reflexión sobre el mismo concepto con el que se había abierto con la obra de autores como Carl Spitteler, Jakob Bosshart, Albin Zollinger o Jakob Schaffner. Pero parece ser que la literatura que se avecina para el siglo XXI va a tener un carácter decididamente diferente: los jóvenes escritores parecen decididos a abrir la literatura suiza a nuevos horizontes y en sus obras se dejan ya ver nuevos temas para un nuevo siglo, nuevos temas para una nueva literatura. 
